\title{
Is birth weight associated with blood pressure among African children and adolescents? A systematic review
}

\author{
S. A. Lule ${ }^{1,2 *}$, A. M. Elliott ${ }^{1,2}$, L. Smeeth ${ }^{1}$ and E. L. Webb ${ }^{1}$ \\ ${ }^{1}$ London School of Hygiene and Tropical Medicine, London, UK \\ ${ }^{2}$ MRC/UVRI Uganda Research Unit, Entebbe, Uganda
}

\begin{abstract}
There is substantial evidence of an inverse association between birth weight and later blood pressure (BP) in populations from high-income countries, but whether this applies in low-income countries, where causes of low birth weight are different, is not certain. Objective: We conducted a review of the evidence on the relationship between birth weight and BP among African children and adolescents. Medline, EMBASE, Global Health and Web of Science databases were searched for publications to October 2016. Papers reporting the relationship between birth weight and BP among African children and adolescents were assessed. Bibliographies were searched for further relevant publications. Selected papers were summarized following the preferred reporting items for systematic review and meta-analysis (PRISMA) guidelines. In total, 16 papers from 13 studies conducted in nine African countries (Nigeria, Republic of Seychelles, Gambia, Democratic Republic of Congo, Cameroon, South Africa, Algeria, Zimbabwe and Angola) were reviewed. Eight studies were cohorts, while five were cross-sectional. The relationship between birth weight and later BP varied with age of the participants. Studies in neonates showed a consistently positive association, while predominantly inverse associations were seen among children, and studies in adolescents were inconsistent. Based on the limited number of studies identified, the relationship between birth weight and later BP may vary with age in African children and adolescents. Not all studies adequately controlled for confounding, notably gender or age. Whether the inverse relationship between birth weight and BP in later life observed in Western settings is also seen in Africa remains unclear.
\end{abstract}

Received 22 August 2017; Revised 16 November 2017; Accepted 18 November 2017; First published online 22 January 2018

Key words: Africa, birth weight, blood pressure, systematic review

\section{Introduction}

A strong geographical correlation between infant mortality (from 1921 to 1925 ) and adult ischaemic heart disease (IHD) mortality (from 1968 to 1978) was observed by Barker and Osmond. ${ }^{1}$ They postulated that factors that increased the risk of death during infancy also increased susceptibility to IHD among those who survived infancy, and later, showed that blood pressure (BP) in adulthood was positively related to placenta weight but inversely associated with birth weight. ${ }^{2}$ They suggested that poor fetal nutrition indicated by intrauterine growth restriction and low birth weight was associated with this increased susceptibility to IHD.

Subsequently, several studies [mainly from high-income countries (HICs)] have investigated the relationship between birth size parameters (e.g. birth weight, head circumference, placenta size) and later cardiovascular disease risk (mainly BP), with birth weight the most widely studied parameter. Results from several of these studies have shown an inverse association between birth weight and BP later in life. ${ }^{3-7}$ A smaller number of studies have reported positive or no association between birth weight and later BP. For example, positive associations have been

${ }^{*}$ Address for correspondence: S. A. Lule, Faculty of Epidemiology and Population Health, London School of Hygiene and Tropical Medicine, London WC1E 7HT, UK.

(Email swaiblule@yahoo.com) reported among UK neonates ${ }^{8}$ and Chinese children, ${ }^{9}$ whereas birth weight was not associated with BP among American adolescents. ${ }^{10}$ The relationship between birth weight and later BP differed by gender among UK adolescents: a negative association was seen in the males but a positive association in the females. ${ }^{11}$ Systematic reviews have reported that, on average, systolic blood pressure (SBP) drops by $2-4 \mathrm{mmHg}$ for every kilogram increase in birth weight. ${ }^{12,13}$ These reviews have predominantly comprised of studies among adults from HICs.

In HICs the prevalence of low birth weight varies between 5 and $8 \% .{ }^{14}$ Low birth weight is more common in Africa (7\% in Nigeria, ${ }^{15} 8 \%$ Uganda, ${ }^{16} 11 \%$ Zambia ${ }^{17} 17 \%$ Zimbabwe and Benin ${ }^{18,19}$ and $28 \%$ Ethiopia $^{18}$ ) and on average African population have lower birth weights when compared with European populations. ${ }^{20}$ In HICs, low birth weight is predominantly due to prematurity (most commonly as a result of maternal smoking in pregnancy ${ }^{21}$ ), whereas, in developing countries, low birth weight for gestational age constitutes most of the low birth weight infants. ${ }^{22}$ The causes of low birth weight differ between rural, tropical Africa and developed or non-tropical settings: for example, malaria (an important cause of low birth weight) is restricted to the tropics ${ }^{23}$ and prophylactic antimalarial drugs in pregnancy reduce the risk of low birth weight. ${ }^{24,25}$ We hypothesized that the relationship between birth weight and BP in African settings might differ from that commonly observed in HICs. 
The role of birth weight in the later development of BP is important to African countries: these have a high burden of malnutrition, ${ }^{26-28}$ low birth weight ${ }^{18,19}$ and raised BP. ${ }^{29-32}$ Early life interventions that reduce maternal malnutrition and extremes of birth weight (both low and high) may thus control childhood BP (before clinical manifestation of disease) and could be vital in the prevention of high BP in adulthood.

The absence of birth weight records for adults in many African countries and the low accuracy of maternally recalled birth weight ${ }^{33}$ limits prospects for studying the relationship between birth weight and BP in adulthood in this setting. However, the emergence of a number of birth cohorts (with birth records) in Africa provides opportunities to investigate the relationship between birth weight and BP among African children and adolescents. Childhood BP predicts BP in early adulthood, ${ }^{34,35}$ thus studies of the relationship between birth weight and BP among children are important in the identification of at-risk groups for targeted interventions early in life. We conducted a qualitative assessment of the direction and consistency of the relationship between birth weight and BP among African children and adolescents using a systematic review of existing literature.

\section{Methods}

A literature search covering publications up to 15 October 2016 with no restriction on start date was performed using Medline, EMBASE, Global Health and Web of Science databases. The search was performed on combinations of the keywords: (hypertension OR blood pressure) AND (birth weight) AND (paediatric OR child OR young people OR youth OR juvenile OR adolescent OR youngster OR pubescent OR teenage OR new-born OR minor OR infant) AND (Africa $\mathrm{OR}$ individual names of countries in Africa).

Original papers on the relationship between birth weight and BP among children and or adolescents, between ages 0 and 19 years and resident in Africa were reviewed. No restrictions on language or publication dates were applied. Publications on children and or adolescents of African ancestry not residing in Africa were excluded. Papers on the same participants were considered as one study. If more than one paper reported on the same participants at the same age, the most complete paper was included in the review. Papers reporting on the same participants were included and reviewed separately if they reported on the relationship between birth weight and BP at different ages. No additional information was sought from authors. Reference lists of the included papers were searched for additional relevant publications.

Search results were exported to Endnote reference management software (Thomson Reuters, version $\times 7$ ) and duplicates removed. Two independent authors (S.L. and E.W.) assessed titles and abstracts for inclusion in the full-text review and then assessed full-text articles for inclusion in the data synthesis. Inconsistencies were discussed and consensus reached at each stage of the selection process.
Data were extracted independently by two authors (S.L. and E.W.) using standardized data extraction sheets on the year of publication, year of birth, location, age of participants, study design, number of participants, exclusion criteria, study aim, mean birth weight, source of birth weight data, BP measurement procedure, mean BP [SBP and, or, diastolic blood pressure (DBP)], relationship between birth weight and BP and how this was assessed, and whether there was adjustment for confounders. Information was recorded as presented in the original publication, except where the overall mean BP or birth weight was missing; in this case, where possible the overall mean was calculated from any stratum-specific means presented. Studies were assessed for selection bias and adjustment for confounding. Meta-analysis was not performed due to diversity in studies included in the review, in terms of their design, analysis, source population and covariates controlled for in the analysis. Guidelines from the preferred reporting items for systematic review and meta-analysis (PRISMA) ${ }^{36}$ were followed.

\section{Results}

A total of 990 published abstracts were retrieved from four databases, of these 366 duplicates were removed, leaving 624 abstracts for review (Fig. 1). Of these, 562 were excluded and of the remaining 62 papers that were subjected to full-text review, 46 were excluded. Of the 46 papers excluded, two papers ${ }^{37,38}$ were duplicates (reported on the same participants at the same age) of one of the included papers. Thus, 16 papers from 13 studies describing, but not necessarily focussing on, the relationship between birth weight and BP were included in the final review and data extraction (Fig. 1).

Of the 16 papers reviewed, six were from West Africa, ${ }^{39-44}$ six Southern Africa, ${ }^{45-50}$ two Central Africa, ${ }^{51,52}$ one East Africa ${ }^{53}$ and one North Africa. ${ }^{54}$ Four papers from Southern Africa were from the same cohort but presented data on BP at different ages of follow-up. ${ }^{46-49}$ Four papers reported results in neonates (0-28 days), ${ }^{41,43,44,51}$ four in children (1-9 years), ${ }^{39,40,49,55}$ four in adolescents (10-19 years) ${ }^{42,47,48,54}$ and four in both children and adolescents. ${ }^{45,46,52,53}$ The papers were published between 1989 and 2016 .

The main characteristics of the reviewed papers are shown in Table 1. Briefly, all papers included both males and females. The number of participants ranged from 157 to 2743 individuals per paper, with five papers reporting on more than 1000 participants. Seven papers had less than 500 participants. Two of the reviewed papers did not present quantitative information on the relationship between birth weight and BP. Eleven of the papers (from eight studies) described results from cohorts, while five papers reported results from cross-sectional studies.

Except for one paper, in which the source of participants was unclear, participants were recruited from schools $(n=5),{ }^{45,50,52-54}$ hospitals $(n=4)^{41,43,44,51}$ and communities ( $n=6$; representing three studies)..$^{40,42,46-49}$ Preterm children were excluded in seven papers: all four of the hospital-based 


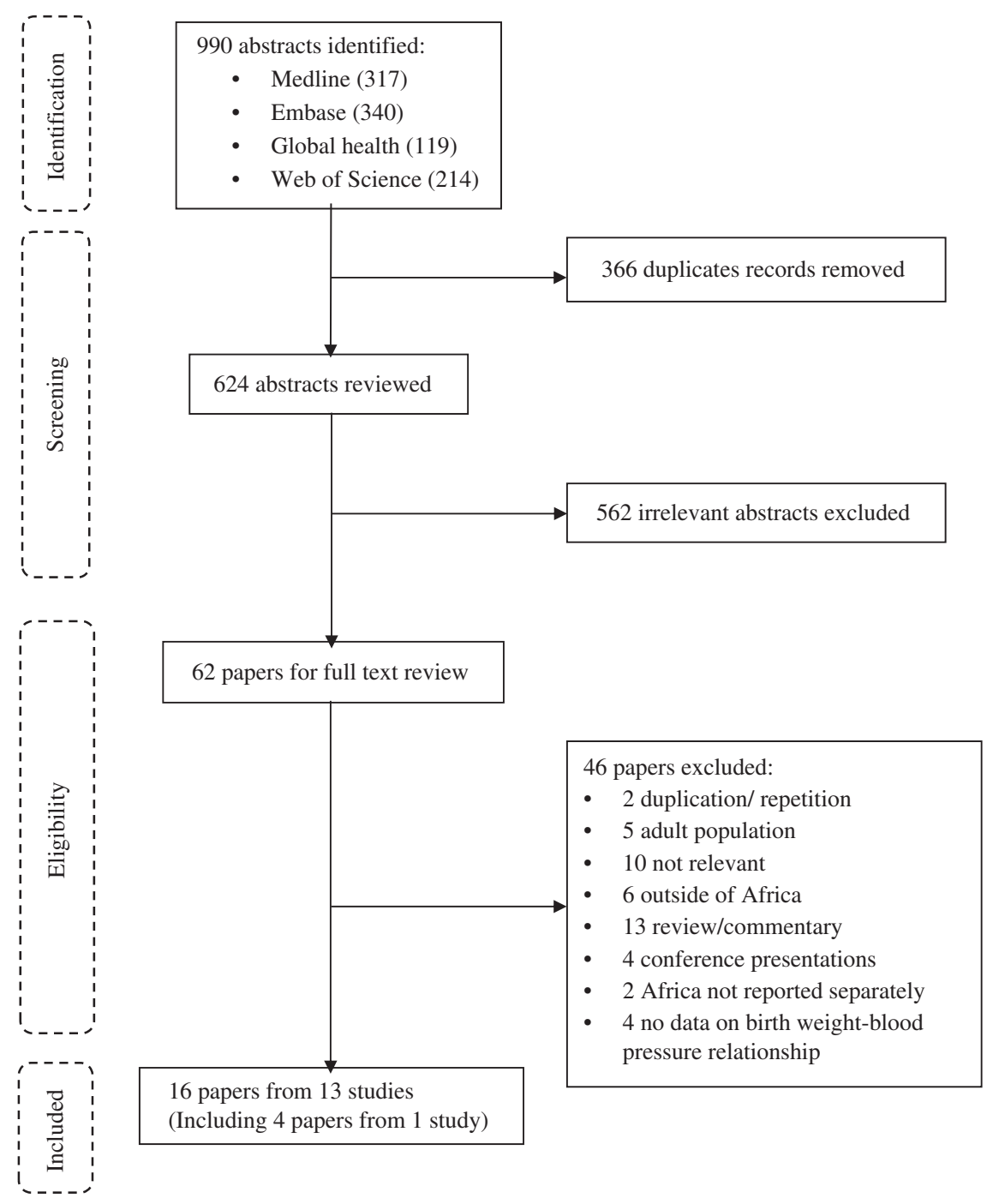

Fig. 1. Flow diagram for systematic review.

studies, ${ }^{41,43,44,51}$ one school-based ${ }^{52}$ and one communitybased. $^{42}$ Twins were excluded in six papers (representing three studies). ${ }^{41,46-48,50}$ Children who were small for gestational age or who weighed $<2.5 \mathrm{~kg}$ at birth were excluded from three papers; one in neonates, ${ }^{44}$ one in children ${ }^{39}$ and one in children and adolescents. ${ }^{52}$ There was variability in the study aims of the papers included: only six described assessing the association between birth weight and BP as one of their main aims. ${ }^{39,50,52-54,56}$

Table 2 summarizes birth weight and BP measurements and values, statistical analysis methods and the relationship between birth weight and BP in the reviewed papers.

\section{Birth weight ascertainment}

Birth weight was either measured and recorded immediately after birth (predominantly in the cohort studies), or extracted from birth or child health card records. In one study, ${ }^{52}$ parentally recalled birth weight was used when birth records were missing (in an unknown number of participants). Mean birth weight varied from 2.4 to $3.4 \mathrm{~kg}$.

\section{BP assessment}

BP procedures were relatively similar across studies. All studies used automated devices, except for one study, which used the sphygmomanometer machine. Eleven papers reported a resting period (from 5 to $20 \mathrm{~min}$ ) before proceeding with measuring the BP. In four studies, measurements were taken on the left arm, whereas in six studies measurements were on the right arm; the remaining studies did not include this information.

BP was measured in triplicates in the majority of papers $(n=12)$ with one paper reporting single measurement, two reporting double measurement and one reporting five measurements. The rest period between consecutive BP measurements varied from 1 to $3 \mathrm{~min}$.

Of the 12 papers that measured BP in triplicate, five used the mean of all three measurements in data analysis, five used the 
Table 1. Description of the studies included in the systematic review

\begin{tabular}{|c|c|c|c|c|c|c|c|c|c|}
\hline Authors & $\begin{array}{c}\text { Year of } \\
\text { publication }\end{array}$ & Year of birth & Place, country & $\begin{array}{l}\text { Age at BP } \\
\text { assessment }\end{array}$ & Study size & Study type & $\begin{array}{l}\text { Source of } \\
\text { subjects }\end{array}$ & Reason for exclusion & Study aim \\
\hline Ayoola & 2011 & NM & Ibadan, Nigeria & $1-3$ days & 436 & Cohort & Hospital & $\begin{array}{l}\text { Preterm, twins, metabolic defects, } \\
\text { congenital abnormalities or sever } \\
\text { birth trauma, babies of women } \\
\text { with HIV, STDs, hypertension } \\
\text { or diabetes }\end{array}$ & $\begin{array}{l}\text { Evaluate the impact of maternal } \\
\text { malaria on new-born BP }\end{array}$ \\
\hline Chiolero & 2011 & 1984-1997 & $\begin{array}{l}\text { Republic of } \\
\text { Seychelles }\end{array}$ & $\begin{array}{l}5.5,9.1,12.5 \\
15.5 \text { years }\end{array}$ & 2743 & Cohort & School & Not mentioned & $\begin{array}{l}\text { Assess the association between } \\
\text { BW, weight change, and } \\
\text { current BP across the entire age } \\
\text { span of childhood and } \\
\text { adolescence }\end{array}$ \\
\hline Hawkesworth & 2009 & 1989-1994 & $\begin{array}{l}\text { West Kiang, } \\
\text { Gambia }\end{array}$ & $11-17$ years & 1267 & Cohort & Community & $\begin{array}{l}\text { Preterm, implausible BP reading, } \\
\text { ambiguity on treatment } \\
\text { allocation }\end{array}$ & $\begin{array}{l}\text { Investigate the effect of maternal } \\
\text { protein-energy } \\
\text { supplementation on BP in } \\
\text { adolescence }\end{array}$ \\
\hline Longo-Mbenza & 1999 & 1980-1991 & Kinshasa, DRC & $\begin{array}{l}5-16 \text { years, } \\
\text { mean } 11 \text { years }\end{array}$ & 2409 & CS & School & $\begin{array}{l}\text { Preterm birth, small for } \\
\text { gestational age }\end{array}$ & $\begin{array}{l}\text { Examine the possible association } \\
\text { between LBW and } \\
\text { hypertension later in life }\end{array}$ \\
\hline Margetts & 1991 & $1980-1988$ & Rural, Gambia & $1-9$ years & 675 & Cohort & Community & Febrile illnesses & $\begin{array}{l}\text { Relate BP levels in children to } \\
\text { their mother's weight in } \\
\text { pregnancy }\end{array}$ \\
\hline Sadoh & 2010 & NM & $\begin{array}{l}\text { Benin City, } \\
\text { Nigeria }\end{array}$ & $1-4$ days & 473 & CS & Hospital & $\begin{array}{l}\text { Preterm, abnormal APGAR score, } \\
\text { congenital abnormality, } \\
\text { admission to neonatal unit, } \\
\text { babies of mothers with pre- } \\
\text { eclampsia or diabetes }\end{array}$ & $\begin{array}{l}\text { Determine the association } \\
\text { between maternal and neonatal } \\
\text { factors with BP at birth }\end{array}$ \\
\hline Salvi & 2010 & $1986-1990$ & Metlili, Algeria & $15-19$ years & 568 & CS & School & $\begin{array}{l}\text { Major disabilities, significant heart } \\
\text { disease, renal or liver disease }\end{array}$ & $\begin{array}{l}\text { Assess the association of current } \\
\text { body weight and birth weight } \\
\text { with BP values in school } \\
\text { children living in Algerian } \\
\text { Sahara }\end{array}$ \\
\hline Law & 2000 & 1989-1993 & $\begin{array}{l}\text { Sagamu } \\
\text { Nigeria }\end{array}$ & $\begin{array}{l}3-6 \text { years, mean } \\
4.4 \text { years }\end{array}$ & 293 & Cohort & NM & Preterm, weighing less than $2.5 \mathrm{~kg}$ & $\begin{array}{l}\text { Determine how reduced fetal } \\
\text { growth is related to raised BP in } \\
\text { countries where chronic } \\
\text { malnutrition is common }\end{array}$ \\
\hline Woelk & 1998 & 1987-1989 & $\begin{array}{l}\text { Harare, } \\
\text { Zimbabwe }\end{array}$ & Mean 6.5 years & 583 & Cohort & School & Twins, not born in Harare & $\begin{array}{l}\text { Determine whether poor uterine } \\
\text { growth may be associated with } \\
\text { increased BP and subsequent } \\
\text { hypertension in adulthood }\end{array}$ \\
\hline
\end{tabular}


Table 1: (Continued)

\begin{tabular}{|c|c|c|c|c|c|c|c|c|c|}
\hline Authors & $\begin{array}{c}\text { Year of } \\
\text { publication }\end{array}$ & Year of birth & Place, country & $\begin{array}{l}\text { Age at BP } \\
\text { assessment }\end{array}$ & Study size & Study type & $\begin{array}{l}\text { Source of } \\
\text { subjects }\end{array}$ & Reason for exclusion & Study aim \\
\hline Silva & 2016 & 2000-2006 & $\begin{array}{l}\text { Luanda, } \\
\text { Angola }\end{array}$ & $\begin{array}{l}7-12 \text { years, } \\
\text { mean } 9.4 \text { years }\end{array}$ & 157 & CS & School & $\begin{array}{l}\text { Not classified as Tanner stage I, } \\
\text { completed } 12 \text { months between } \\
\text { recruitment and examinations, } \\
\text { high BP, obesity }\end{array}$ & $\begin{array}{l}\text { Determine the factors associated } \\
\text { with pulse wave velocity values } \\
\text { and propose preliminary } \\
\text { reference values in pre-pubertal } \\
\text { Angolan school children }\end{array}$ \\
\hline Nwokoye & 2015 & NM & Enugu, Nigeria & $1-2$ days & 310 & CS & Hospital & $\begin{array}{l}\text { Birth asphyxia, preterm, postterm, } \\
\text { sick babies, not weighing } \\
2.5-4.0 \mathrm{~kg} \text {, babies of mothers } \\
\text { on antihypertensive drugs or } \\
\text { illicit drugs }\end{array}$ & $\begin{array}{l}\text { Determine BP values in } \\
\text { apparently healthy term new } \\
\text { borns in the first } 48 \mathrm{~h} \text { of life } \\
\text { and evaluate the factors } \\
\text { affecting BP at birth }\end{array}$ \\
\hline Youmbissi & 1989 & NM & $\begin{array}{l}\text { Yaounde, } \\
\text { Cameroon }\end{array}$ & At birth & 202 & Cohort & Hospital & $\begin{array}{l}\text { Preterm, sick neonates, babies of } \\
\text { mothers on diuretics or } \\
\text { antihypertensive therapy during } \\
\text { pregnancy or labour }\end{array}$ & $\begin{array}{l}\text { Evaluate SBP variations in new } \\
\text { borns }\end{array}$ \\
\hline Levitt $^{a}$ & 1999 & 1990 & $\begin{array}{l}\text { Soweto, South } \\
\text { Africa }\end{array}$ & 5 years & 818 & Cohort & Community & Twins & $\begin{array}{l}\text { Examine the relationship between } \\
\text { BW and BP at } 5 \text { years in a } \\
\text { cohort of South African } \\
\text { children }\end{array}$ \\
\hline Kagura $^{a}$ & 2016 & 1990 & $\begin{array}{l}\text { Soweto South } \\
\text { Africa }\end{array}$ & $\begin{array}{l}5,8,10,13,14, \\
16 \text { and } 18 \text { years }\end{array}$ & 1937 & Cohort & Community & $\begin{array}{l}\text { Twins, non-black, pregnancy } \\
\text { during adolescence }\end{array}$ & $\begin{array}{l}\text { Examine the association between } \\
\text { early growth and BP } \\
\text { trajectories and assess the } \\
\text { influence of height on the } \\
\text { association between early } \\
\text { growth and BP trajectories }\end{array}$ \\
\hline Griffiths $^{a}$ & 2012 & 1990 & $\begin{array}{l}\text { Soweto, South } \\
\text { Africa }\end{array}$ & 16 years & 358 & Cohort & Community & Twins, non-black & $\begin{array}{l}\text { Understand the relationship } \\
\text { between household and } \\
\text { neighbourhood SES with SBP }\end{array}$ \\
\hline Adair $^{\mathrm{a}}$ & 2013 & 1990 & $\begin{array}{l}\text { Soweto, South } \\
\text { Africa }\end{array}$ & 18 years & 1222 & Cohort & Community & Twins & $\begin{array}{l}\text { Investigate how linear growth and } \\
\text { weight gain relative to linear } \\
\text { growth in childhood and } \\
\text { adulthood are related to health } \\
\text { and human capital outcomes in } \\
\text { young adults }\end{array}$ \\
\hline
\end{tabular}

BP, blood pressure; BW, birth weight; DRC, Democratic Republic of Congo; CS, cross-sectional study; LBW, low birth weight; NM, not mentioned; SBP, systolic blood pressure; SES, socioeconomic status.

${ }^{\mathrm{a}}$ Papers from the same cohort (Birth to Twenty cohort). 
Table 2. Main results from the studies included in the systematic review

\begin{tabular}{|c|c|c|c|c|c|c|}
\hline Authors & Mean BW & $\begin{array}{c}\text { Source of BW } \\
\text { data }\end{array}$ & Mean BP $(\mathrm{mmHg})$ & Procedure for BP measurement & Relationship between BW and BP & Adjusting factors \\
\hline Ayoola & $2.9 \mathrm{~kg}$ & $\begin{array}{l}\text { Hospital birth } \\
\text { records }\end{array}$ & $\begin{array}{l}\mathrm{SBP}=71.0 \\
\mathrm{DBP}=36.1\end{array}$ & $\begin{array}{l}\text { With the child comfortable on the mother's lap for } 5 \text { min or } \\
\text { asleep, three BP measurements with a minute's interval } \\
\text { between successive measurements were taken on the left arm } \\
\text { using a Datascope monitor. Mean of the last two readings } \\
\text { was used for analysis }\end{array}$ & $\begin{array}{l}\text { Positive BW-BP association. SBP increased } \\
\text { by } 8.35 \mathrm{mmHg} / \mathrm{kg} \text { increase in BW }(95 \% \\
\text { CI: } 4.36,12.35, P<0.001) \text {. DBP } \\
\text { increased by } 3.07 \mathrm{mmHg} / \mathrm{kg} \text { increase in } \\
\text { BW }(95 \% \mathrm{CI}: 0.26,5.88, P=0.032)\end{array}$ & $\begin{array}{l}\text { Gestational age, baby } \\
\text { length, maternal } \\
\text { malaria, age, weight, } \\
\text { height, BP, } \\
\text { gravidity, antenatal } \\
\text { visits }\end{array}$ \\
\hline Chiolero & $3.1 \mathrm{~kg}$ & $\begin{array}{l}\text { Medical } \\
\text { records }\end{array}$ & $\begin{array}{l}\text { Could not be } \\
\text { determined }\end{array}$ & $\begin{array}{l}\text { After 5-min rest, two BP measurements were taken on the right } \\
\text { arm with a minute's interval between each using automated } \\
\text { devices (OmronM5; Omron, UK). Mean of the two } \\
\text { measurements was used for analysis }\end{array}$ & $\begin{array}{l}\text { No overall BW-BP association. BW was } \\
\text { not associated with SBP or with DBP, } \\
\text { with exception of girls at } 12.5 \text { years } \\
\text { among whom BW was inversely } \\
\text { associated with SBP }(\beta=0.9,95 \% \mathrm{CI} \text { : } \\
-1.6,-0.1, P=0.026) \text { and DBP } \\
(\beta=-0.7,95 \% \text { CI: }-1.3,-0.1, \\
P=0.028)\end{array}$ & Current weight \\
\hline Hawkesworth & $2.9 \mathrm{~kg}$ & Birth records & $\begin{array}{l}\mathrm{SBP}=110.5 \\
\mathrm{DBP}=64.7\end{array}$ & $\begin{array}{l}\text { Measured in triplicate using the automated Omron } 7051 \mathrm{~T} \\
\text { device (Omron), following manufacturer's instruction. } \\
\text { Mean of the three measurements was used for analysis }\end{array}$ & $\begin{array}{l}\text { No association between BW and BP, } \\
\begin{aligned} \beta & =-0.001,95 \% \mathrm{CI}:-0.002,0.000 \\
P & =0.06\end{aligned}\end{array}$ & $\begin{array}{l}\text { Age, current body size, } \\
\text { sex, gestational age, } \\
\text { birth season }\end{array}$ \\
\hline Longo-Mbenza & $2.4 \mathrm{~kg}$ & $\begin{array}{l}\text { Parental recall, } \\
\text { medical } \\
\text { records }\end{array}$ & $\begin{array}{l}\text { Could not be } \\
\text { determined }\end{array}$ & $\begin{array}{l}\text { With child in a sitting position and relaxed for } 20 \text { min, five BP } \\
\text { measurements were obtained using an automatic device } \\
\text { (HEM-705 CP; Omron, Tokyo, Japan). Not clear which } \\
\text { measurements were used in the analysis }\end{array}$ & $\begin{array}{l}\text { BW inversely correlated with BP. With } \\
\quad r=-0.1, P<0.001 \text { for SBP and } \\
r=-0.1, P<0.05 \text { for DBP. LBW had } \\
\text { twice the odds of hypertension } \\
\text { OR }=2.0,95 \% \text { CI: } 0.9,8.2, P<0.01 \\
\text { for SBP and OR }=2.3,95 \% \text { CI: } 0.6 \\
-11.5, P<0.01 \text { for } \mathrm{DBP}^{\mathrm{a}}\end{array}$ & NM \\
\hline Margetts & $3.0 \mathrm{~kg}$ & Birth records & $\begin{array}{c}1 \text { year: } \mathrm{SBP}=89.3 \\
\mathrm{DBP}=56.2 \\
9 \text { years: } \mathrm{SBP}=102.7 \\
\mathrm{DBP}=63.9\end{array}$ & $\begin{array}{l}\text { After } 5 \text { min in a sitting position or on the mother's knee in the } \\
\text { young children, BP was measured twice on the right arm } \\
\text { using an automated device (Dinamap model: } 18465 \mathrm{X} \text { ). Not } \\
\text { clear which measurements were used in the analysis }\end{array}$ & $\mathrm{BW}$ not associated with SBP at any age & NM \\
\hline Sadoh & $3.2 \mathrm{~kg}$ & $\begin{array}{l}\text { Hospital birth } \\
\text { records }\end{array}$ & $\mathrm{SBP}=69.2$ & $\begin{array}{l}\text { Measured } 1 \mathrm{~h} \text { after feeds between } 11: 00 \text { and } 13: 30 \text { using a } \\
\text { Dinamap } 8100 \text { monitor (Critikon, Tampa Fla) device, when } \\
\text { the baby was asleep or awake and calm. Three BP } \\
\text { measurements were obtained within } 3 \text { min of each other on } \\
\text { the right arm. Mean of three BP readings was used in the } \\
\text { analysis }\end{array}$ & $\begin{array}{l}\text { Positive BW-BP association. BW was } \\
\text { correlated with SBP }(r=0.235 \\
P=0.001), \text { SBP rose by } 3.61 \mathrm{mmHg} / \\
0.5 \mathrm{~kg} \text { increase in birth weight }\end{array}$ & NM \\
\hline Salvi & $3.4 \mathrm{~kg}$ & $\begin{array}{l}\text { Obstetric } \\
\text { records in } \\
\text { local hospitals }\end{array}$ & $\begin{array}{l}\mathrm{SBP}=118.1 \\
\mathrm{DBP}=69.9\end{array}$ & $\begin{array}{l}\text { Three BP measurements with 3-min interval between successive } \\
\text { measurements were taken on the left arm, after 10-min rest } \\
\text { in a sitting position. An automated oscillmetric device } \\
\text { (Omron 705 T; Omron) was used. Average of the three } \\
\text { measurements was used for the analysis }\end{array}$ & No correlation between BW and SBP & $\begin{array}{l}\text { Multivariate analysis } \\
\text { was not done }\end{array}$ \\
\hline Law & $3.2 \mathrm{~kg}$ & Birth records & $\mathrm{SBP}=101.6$ & $\begin{array}{l}\text { After 5-min rest, three BP measurements were taken on the left } \\
\text { arm using automated BP machines (Dinamap model } 8100 \text { ) } \\
\text { with a 1-min interval between consecutive measurements. } \\
\text { Mean of the three measurements was used for the analysis }\end{array}$ & $\begin{array}{l}\text { No association between BW and SBP } \\
\quad(\beta=0.4,95 \% \text { CI: }-2.1,2.9)\end{array}$ & $\begin{array}{l}\text { Gender, observer, } \\
\text { child's status, (crying } \\
\text { or not) current } \\
\text { weight and cuff size }\end{array}$ \\
\hline
\end{tabular}




\begin{tabular}{|c|c|c|c|c|c|c|}
\hline Authors & Mean BW & $\begin{array}{c}\text { Source of BW } \\
\text { data }\end{array}$ & Mean BP $(\mathrm{mmHg})$ & Procedure for BP measurement & Relationship between $\mathrm{BW}$ and $\mathrm{BP}$ & Adjusting factors \\
\hline Woelk & $3.0 \mathrm{~kg}$ & Birth records & $\begin{array}{l}\mathrm{SBP}=108.3 \\
\mathrm{DBP}=62.1\end{array}$ & $\begin{array}{l}\text { With child sitting quietly, BP was measured in the morning on } \\
\text { the right arm. Three measurements were taken 2-min apart } \\
\text { using a Dinamap model } 8100 \mathrm{BP} \text {. The average of the last two } \\
\text { BP readings was used for analysis }\end{array}$ & $\begin{array}{l}\text { Inverse relationship between BW and SBP. } \\
\text { SBP rose by } 1.73 \mathrm{mmHg} / \mathrm{kg} \text { decrease in } \\
\text { BW ( } 95 \% \text { CI: } 0.18,3.28), P=0.0286 \text {. } \\
\text { No association between } \mathrm{BW} \text { and DBP } \\
(\beta=-1.06,95 \% \text { CI: }-2.57,0.45)\end{array}$ & Current weight \\
\hline Silva & $3.2 \mathrm{~kg}$ & - & $\begin{array}{l}\mathrm{SBP}=102 \\
\mathrm{DBP}=62\end{array}$ & $\begin{array}{l}\text { After resting for } 5-10 \text { min in a sitting position, three } \\
\text { consecutive BP measurements were taken on the left arm } \\
\text { with a 2-min interval using an automatic } \\
\text { sphygmomanometer (model HEM- } 742 \text {; OMRON, } \\
\text { Nanjing, China). Mean of last two readings was used for } \\
\text { analysis }\end{array}$ & $\begin{array}{l}\text { No correlation between BW and SBP, } \\
r=-0.016 \text {, weak correction between } \\
\text { DBP and BW } r=0.09, P<0.05 \text {. } \\
\text { Categorizing birth weight into four } \\
\text { groups, no association between birth } \\
\text { weight category and SBP }(P=0.991) \text { or } \\
\text { DBP }(P=0.059)\end{array}$ & NM \\
\hline Nwokoye $^{\text {b }}$ & $2.5-4.0 \mathrm{~kg}$ & $\begin{array}{l}\text { Hospital birth } \\
\text { records }\end{array}$ & $\begin{array}{l}\text { Day } 1, \mathrm{SBP}=63.3 \\
\mathrm{DBP}=36.8 \\
\text { Day } 2, \mathrm{SBP}=65.6 \\
\mathrm{DBP}=40.0\end{array}$ & $\begin{array}{l}\text { After } 10-15 \text {-min rest, a single BP measurement was taken on } \\
\text { the right arm when the infant was awake and quiet or asleep } \\
\text { and in spine position using an oscillometic machine } \\
\text { (Dinamap 8100) }\end{array}$ & $\begin{array}{l}\text { Positive correlation between BW and SBP, } \\
r=0.37, P<0.01 \text { between } 0-24 \mathrm{~h} \text { and } \\
r=0.29, P<0.01 \text { between } 25-48 \mathrm{~h} \text {. No } \\
\text { correlation between BW and DBP }\end{array}$ & NM \\
\hline Youmbissi & $3.2 \mathrm{~kg}$ & $\begin{array}{l}\text { Hospital birth } \\
\text { records }\end{array}$ & $\mathrm{SBP}=65.1$ & $\begin{array}{l}\text { Measured in the morning, on the right arm of a quiet and awake } \\
\text { child. Three measurements were taken using a zero } \\
\text { sphygmomanometer. Average of the three measurements } \\
\text { used for the analysis }\end{array}$ & $\begin{array}{l}\text { No association between BW and SBP, } \\
\quad r=0.12\end{array}$ & NM \\
\hline Levitt $^{c}$ & $3.1 \mathrm{~kg}$ & Birth records & $\begin{array}{l}\mathrm{SBP}=108.0 \\
\mathrm{DBP}=62.6\end{array}$ & $\begin{array}{l}\text { After 10-min rest, BP was measured in triplicate using a } \\
\text { Dinamap vital signs Monitor (1846SX). The lowest DBP } \\
\text { with its matching SBP were used in the analysis }\end{array}$ & $\begin{array}{l}\text { Inverse association between BW and SBP } \\
(r=-0.05, P<0.001), \mathrm{SBP} \text { fell by } \\
3.4 \mathrm{mmHg},(95 \% \mathrm{CI}: 1.4,5.3) / \mathrm{kg} \\
\text { increase in BW. No association between } \\
\text { BW and DBP }\end{array}$ & $\begin{array}{l}\text { Current weight and } \\
\text { height }\end{array}$ \\
\hline Kagura $^{c}$ & $3.1 \mathrm{~kg}$ & Birth records & $\begin{array}{l}\text { Could not be } \\
\text { determined }\end{array}$ & $\begin{array}{l}\text { After 5-min rest, BP was measured in triplicate with 2-min } \\
\text { intervals between successive measurements, using the } \\
\text { Dinamap Signs monitor } 1846 \mathrm{XX} \text { (Critikon) at } 5 \text { years and } \\
\text { Omron M6 (Omron) at } 8-18 \text { years. Not clear which } \\
\text { measurements were used in the analysis }\end{array}$ & $\begin{array}{l}\text { Inverse association between BW and } \\
\text { middle BP trajectory among boys } \\
(\mathrm{OR}=0.75,95 \% \text { CI: } 0.58,0.96 \\
P=0.0223) \text {. No other associations seen }\end{array}$ & $\begin{array}{l}\text { Height, SES, maternal } \\
\text { age, parity and } \\
\text { gestational age }\end{array}$ \\
\hline Griffiths $^{c}$ & $3.1 \mathrm{~kg}$ & Birth records & $\mathrm{SBP}=114.8$ & $\begin{array}{l}\text { With participants in a sitting position, three measurements were } \\
\text { taken using a digital (Omron M6; Omron) device with a rest } \\
\text { of several minutes between successive measurements. The } \\
\text { average of the last two measurements was used for analysis }\end{array}$ & $\begin{array}{l}\text { Inverse association between } \mathrm{BW} \text { and } \mathrm{SBP} \\
\text { among boys }(\beta=-0.003, P<0.1) \text {. No } \\
\text { association between } \mathrm{BW} \text { and SBP } \\
\text { among girls }\end{array}$ & Current height \\
\hline Adair ${ }^{c}$ & $3.1 \mathrm{~kg}$ & $\begin{array}{l}\text { Hospital birth } \\
\text { records }\end{array}$ & $\begin{array}{l}\mathrm{SBP}=117.5 \\
\mathrm{DBP}=71.4\end{array}$ & $\begin{array}{l}\text { After 5-10-min rest, three BP measurements were taken using a } \\
\text { digital device (Omron M6). The average of the last two } \\
\text { measurements was used for analysis }\end{array}$ & $\begin{array}{l}\text { No association between BW and BP. For } \\
\text { SBP } \beta=0.05,95 \% \text { CI: }-0.79,0.90 \text { in } \\
\text { males and } 0.08(-0.69,0.84) \text { in females, } \\
\text { and for DBP } \beta=-0.05(-0.78,0.68) \\
\text { among males and } 0.17(-0.51,0.85) \text { in } \\
\text { females }\end{array}$ & NM \\
\hline
\end{tabular}


mean of the last two measurements, one used the lowest DBP (with matching SBP) and one was unclear. For the paper where $\mathrm{BP}$ was measured five times and one of the two papers where $\mathrm{BP}$ was measured in duplicate, it was unclear which measurements or combination thereof were used for data analysis. In the other paper where BP was measured in duplicate, the mean of the two measurements was used in the analysis.

Among neonates, mean SBP varied from 65.1 to $71.0 \mathrm{mmHg}$ and in children and adolescents from 89.3 to $118.1 \mathrm{mmHg}$. Mean DBP varied from 36.1 to $63.9 \mathrm{mmHg}$ in neonates and between 56.2 and $71.4 \mathrm{mmHg}$ among children and adolescents. Mean SBP and DBP generally increased with age over the course of childhood and adolescence, this was especially apparent in the four papers that reported results from the same cohort study at different ages.

\section{Birth weight and BP relationship}

The relationship between birth weight and SBP varied across papers; seven papers reported no association, ${ }^{39,40,42,45,47,51,54}$ six an inverse association ${ }^{46,48-50,52,53}$ and three a positive association. ${ }^{41,43,44}$ Among the neonates, three out of four papers reported a positive association while one reported no association. Of the four papers in children, two reported inverse associations and two no association. The papers on children and adolescents predominately found inverse associations (three out of four papers) while among adolescents, three papers found no association and one an inverse association.

Of the seven papers with participant size less than 500 individuals, ${ }^{39,41,43-45,48,51}$ three papers reported no association between birth weight and SBP, one an inverse association and three a positive association. ${ }^{43-45,51}$ Studies with larger participant sizes (greater than 500 individuals) were more likely to report inverse associations. Of the nine studies with participant size over 500, three reported no association between birth weight and SBP, five an inverse association and one reported a positive association.

In three of the six papers reporting inverse associations, ${ }^{46,48,53}$ analyses were conducted at different ages and, or, separately for males and females, with inverse associations only seen among particular subgroups (girls at 12.5 years, ${ }^{53}$ boys only ${ }^{46,48}$ ) and analyses from other subgroups showing no evidence of association.

Analysis approaches used to assess the relationship between birth weight and BP were diverse, varying from simple correlation analysis with no adjustment for potential confounders, to more complex group-based trajectory modelling approaches. Multivariable analysis, adjusting for potential confounder(s) [often including age, sex or body size (weight or height)] was conducted in eight papers, of these five reported an inverse association, two no association and one a positive association. In comparison, of the eight papers that did not undertake adjustment for confounders, one reported an inverse association between birth weight and SBP, five reported no association and two a positive association.
The relationship between birth weight and DBP was described in eight papers; a positive association was seen in two papers, inverse association in two papers and no association was reported in four papers. Of these eight papers, two were in neonates, two in children, three in children and adolescents, and one in adolescents only.

\section{Discussion}

Overall, this systematic review of existing literature showed varied results. We identified 16 papers from 13 studies addressing the question of whether the inverse relationship between birth weight and BP in later life seen in Western settings is also present in Africa. The relatively small number of studies and their heterogeneity in design and analysis prohibits definitive conclusions. However, we found some evidence to suggest that the relationship between birth weight and SBP in Africa varies with the participants' age: positive associations were seen in neonates and inverse associations mainly in children. Among adolescents, the relationship was either inverse or showed no evidence of association. Only a few papers reported on the relationship between birth weight and DBP, with most papers reporting no relationship between birth weight and DBP.

This review supports an earlier review by Law ${ }^{12}$ that did not include any of the papers reviewed herein (only two of the papers included in the present review had been published at the time of the Law review, and of these Margetts et al. ${ }^{40}$ was excluded for missing quantitative information while Youmbissi et al. ${ }^{51}$ was not mentioned). The Law review reported inconsistencies in the relationship between birth weight and BP, especially among adolescents. Generally, inverse associations were among children and positive associations in neonates, inconsistencies could be due to differences in age, sample sizes and statistical analysis approaches. Interestingly, results from the cohort studies included in our review, that measured BP at more than one-time point (different ages), did not show evidence of increasing strength of the association between birth weight and BP with age as reported by the earlier review. ${ }^{12}$ Studies in neonates consisted mainly of less than 500 participants and reported positive associations. Studies with smaller participant numbers are more likely to be underpowered to detect real associations, but also to produce spurious positive or negative associations. ${ }^{57}$ However, this may not be a factor for the results among neonates, which are consistent.

The relationship between birth weight and BP among adolescents has been reported in previous reviews as either inconsistent ${ }^{12}$ or inverse with smaller effects than observed among (prepubescent) children. ${ }^{13}$ Similarly, this review found an inconsistent relationship between birth weight and BP among adolescents, while the relationship among younger children was generally inverse. The positive relationship observed in neonates is as expected, with the duration between birth and BP assessment too short to allow for any impact of subsequent weight trajectory. Explanations for the changing relationships 
among children and adolescents are uncertain, but could possibly relate to different growth patterns, for example, catch-up growth among those of low birth weight, and hormonal changes occurring at adolescence. ${ }^{12,58}$

Adjustment for possible confounding factors varied and was often incomplete. Consistent with previous reviews, which mainly included papers from HICs, studies adjusting for current body size (weight and, or, height) were more likely to report an inverse relationship ${ }^{59,60}$ than those that did not make such an adjustment. Adjusting for current size has been noted to lead to a stronger inverse relationship between birth weight and BP compared with results without such adjustments. ${ }^{6}$ In most of the reviewed papers, that reported estimates adjusted for current size, unadjusted estimates for the effect of birth weight on BP were not reported. Therefore, we were unable to establish whether adjusting for current weight leads to stronger inverse relationships in these populations. The interpretation of findings adjusting for current weight is complex ${ }^{6,59}$ because current weight may be seen as a confounder or mediator of the effect of birth weight on BP. ${ }^{6}$

Several mechanisms such as obesity, salt-sensitivity, reninangiotensin system and endothelial activation are important in the pathophysiology of hypertension. None of the reviewed papers investigated the role of these factors and their impact on BP. Recent evidence suggests that the relationship between birth weight and BP could be U shaped ${ }^{61}$ highlighting the importance of both reduced and excessive nutrition in utero. It was not possible to examine this hypothesis from the papers reviewed. Compared with birth weight, other measures such as birth body mass index or pendular index may more accurately reflect the birth size, but none of the studies reviewed included these measures.

Studies were subject to selection bias as individuals most likely to be low birth weight (such as preterm and twins) were excluded in many studies. This could have led to an underestimation of the effect of birth weight on BP. Furthermore, characteristics [such as maternal hypertension, parasitic infections, socioeconomic status (past or current)] that influence birth weight and may also be associated with BP in offspring were not adjusted for in these studies. Hence, estimates were subject to residual confounding. It remains uncertain what role (if any) such factors have in the relationship between birth weight and subsequent BP in children or adolescents.

Inconsistencies seen between reviewed papers are less likely to be due to differences in BP measurement procedures, as studies followed a similar approach. For nearly all papers, there was an initial rest period (before starting BP procedure) and between successive BP measurements, automated devices were used and analysis was based on the average of two or three measurements. In the majority of studies, early life information including birth weight was prospectively collected thus studies were less prone to misclassification and recall bias.

Generally, studies reviewed came from all the African regions but with a strong representation of West Africa and Southern Africa. East Africa and North Africa had the least number of papers (one each) reviewed. The only paper from East Africa reported on an island population, thus there were no results on the mainland population of East Africa.

In conclusion, relatively few studies have investigated the relationship between birth weight and BP later in life in Africa. The relationship between birth weight and BP varied depending on the age of the participants. Our review emphasizes the need for larger studies on the relationship between birth weight and later BP from Africa, applying appropriate control of potential confounding factors. Accumulating evidence on raised BP in Africa and understanding the impact of growth in early life and of prenatal exposures on BP later in life is key in identifying at-risk groups and developing early life interventions to reduce BP risk in later life.

\section{Acknowledgements}

None.

\section{Ethical Standards}

Review was based on published manuscripts thus ethical approvals were not required.

\section{Financial Support}

This work was supported with funding from the Commonwealth Scholarship Commission (S.L., PhD funding at the LSHTM); the Wellcome Trust (A.E., grant number 095778) (L.S., grant number 098504/Z/12/Z); and the UK Medical Research Council (E.W., grant number MR/K012126/1).

\section{Conflicts of Interest}

None.

\section{References}

1. Barker DJ, Osmond C. Infant mortality, childhood nutrition, and ischaemic heart disease in England and Wales. Lancet. 1986; 1, 1077-1081.

2. Barker DJ, Bull AR, Osmond C, Simmonds SJ. Fetal and placental size and risk of hypertension in adult life. BMJ. 1990; 301, 259-262.

3. Schack-Nielsen L, Holst C, Sorensen TI. Blood pressure in relation to relative weight at birth through childhood and youth in obese and non-obese adult men. Int J Obes Relat Metab Disord. 2002; 26, 1539-1546.

4. Adair LS, Martorell R, Stein AD, et al. Size at birth, weight gain in infancy and childhood, and adult blood pressure in 5 low- and middle-income-country cohorts: when does weight gain matter? Am J Clin Nutr. 2009; 89, 1383-1392.

5. Law CM, de Swiet M, Osmond C, et al. Initiation of hypertension in utero and its amplification throughout life. BMJ. 1993; 306, 24-27.

6. Blake KV, Gurrin LC, Evans SF, et al. Adjustment for current weight and the relationship between birth weight and blood pressure in childhood. J Hypertens. 2000; 18, 1007-1012. 
7. Pharoah PO, Stevenson CJ, West CR. Association of blood pressure in adolescence with birthweight. Arch Dis Child Fetal Neonatal Ed. 1998; 79, F114-F118.

8. O'Sullivan MJ, Kearney PJ, Crowley MJ. The influence of some perinatal variables on neonatal blood pressure. Acta Paediatr. 1996; 85, 849-853.

9. Perng W, Rifas-Shiman SL, Kramer MS, et al. Early weight gain, linear growth, and mid-childhood blood pressure: a prospective study in project viva. Hypertension. 2016; 67, 301-308.

10. Falkner B, Hulman S, Kushner H. Effect of birth weight on blood pressure and body size in early adolescence. Hypertension. 2004; 43, 203-207.

11. Macintyre S, Watt G, West P, Ecob R. Correlates of blood pressure in 15 year olds in the west of Scotland. J Epidemiol Community Health. 1991; 45, 143-147.

12. Law CM, Shiell AW. Is blood pressure inversely related to birth weight? The strength of evidence from a systematic review of the literature. J Hypertens. 1996; 14, 935-941.

13. Huxley RR, Shiell AW, Law CM. The role of size at birth and postnatal catch-up growth in determining systolic blood pressure: a systematic review of the literature. J Hypertens. 2000; 18, 815-831.

14. Kim D, Saada A. The social determinants of infant mortality and birth outcomes in Western developed nations: a cross-country systematic review. Int J Environ Res Public Health. 2013; 10, 2296-2335.

15. Dahlui M, Azahar N, Oche OM, Aziz NA. Risk factors for low birth weight in Nigeria: evidence from the 2013 Nigeria Demographic and Health Survey. Glob Health Action. 2016; 9, 28822.

16. Ndibazza J, Muhangi L, Akishule D, et al. Effects of deworming during pregnancy on maternal and perinatal outcomes in Entebbe, Uganda: a randomized controlled trial. Clin Infect Dis. 2010; 50, 531-540.

17. Chibwesha CJ, Zanolini A, Smid M, et al. Predictors and outcomes of low birth weight in Lusaka, Zambia. Int J Gynaecol Obstet. 2016; 134, 309-314.

18. Assefa N, Berhane Y, Worku A. Wealth status, mid upper arm circumference (MUAC) and antenatal care (ANC) are determinants for low birth weight in Kersa, Ethiopia. PloS One. 2012; 7, e39957.

19. Denoeud L, Fievet N, Aubouy A, et al. Is chloroquine chemoprophylaxis still effective to prevent low birth weight? Results of a study in Benin. Malar J. 2007; 6, 27.

20. Shiono PH, Klebanoff MA, Graubard BI, Berendes HW, Rhoads GG. Birth weight among women of different ethnic groups. JAMA. 1986; 255, 48-52.

21. Delnord M, Blondel B, Zeitlin J. What contributes to disparities in the preterm birth rate in European countries? Curr Opin Obstet Gynecol. 2015; 27, 133-142.

22. Garner P, Kramer MS, Chalmers I. Might efforts to increase birthweight in undernourished women do more harm than good? Lancet. 1992; 340, 1021-1023.

23. Shulman CE, Dorman EK. Importance and prevention of malaria in pregnancy. Trans R Soc Trop Med Hyg. 2003; 97, 30-35.

24. Muanda FT, Chaabane S, Boukhris T, et al. Antimalarial drugs for preventing malaria during pregnancy and the risk of low birth weight: a systematic review and meta-analysis of randomized and quasi-randomized trials. BMC Med. 2015; 13, 193.

25. Cot M, Le Hesran JY, Miailhes P, et al. Increase of birth weight following chloroquine chemoprophylaxis during the first pregnancy: results of a randomized trial in Cameroon. Am J Trop Med Hyg. 1995; 53, 581-585.

26. Akombi BJ, Agho KE, Merom D, Renzaho AM, Hall JJ. Child malnutrition in sub-Saharan Africa: a meta-analysis of demographic and health surveys (2006-2016). PloS One. 2017; 12, e0177338.

27. Vorster HH, Kruger A. Poverty, malnutrition, underdevelopment and cardiovascular disease: a South African perspective. Cardiovasc J Afr. 2007; 18, 321-324.

28. Benzekri NA, Sambou J, Diaw B, et al. High prevalence of severe food insecurity and malnutrition among HIV-infected adults in Senegal, West Africa. PloS One. 2015; 10, e0141819.

29. Guwatudde D, Mutungi G, Wesonga R, et al. The epidemiology of hypertension in Uganda: findings from the national noncommunicable diseases risk factor survey. PloS One. 2015; 10, e0138991.

30. Kavishe B, Biraro S, Baisley K, et al. High prevalence of hypertension and of risk factors for non-communicable diseases (NCDs): a population based cross-sectional survey of NCDS and HIV infection in Northwestern Tanzania and Southern Uganda. BMC Med. 2015; 13, 126.

31. Ataklte F, Erqou S, Kaptoge S, et al. Burden of undiagnosed hypertension in sub-Saharan Africa: a systematic review and metaanalysis. Hypertension. 2015; 65, 291-298.

32. Reddy SP, Mbewu AD. The Implications of the Developmental Origins of Health and Disease on Public Health Policy and Health Promotion in South Africa. Parthasarathy S, ed. Healthcare. 2016; 4, 83.

33. Lule SA, Webb EL, Ndibazza J, et al. Maternal recall of birthweight and birth size in Entebbe, Uganda. Trop Med Int Health. 2012; 17, 1465-1469.

34. Kagura J, Adair LS, Musa MG, Pettifor JM, Norris SA. Blood pressure tracking in urban black South African children: Birth to Twenty cohort. BMC Pediatr. 2015; 15, 78.

35. Hao G, Wang X, Treiber FA, et al. Blood pressure trajectories from childhood to young adulthood associated with cardiovascular risk: results from the 23-year longitudinal Georgia stress and heart study. Hypertension. 2017; 69, 435-442.

36. Moher D, Shamseer L, Clarke M, et al. Preferred reporting items for systematic review and meta-analysis protocols (PRISMA-P) 2015 statement. Syst Rev. 2015; 4, 1.

37. Steyn K, de Wet T, Richter L, et al. Cardiovascular disease risk factors in 5-year-old urban South African children - the birth to ten study. S Afr Med J. 2000; 90, 719-726.

38. Sadoh WE, Ibhanesebhor SE. Oscillometric blood pressure reference values of African full-term neonates in their first days postpartum. Cardiovasc J Afr. 2009; 20, 344-347.

39. Law CM, Egger P, Dada O, et al. Body size at birth and blood pressure among children in developing countries. Int J Epidemiol. 2000; 30, 52-57.

40. Margetts BM, Rowland MG, Foord FA, et al. The relation of maternal weight to the blood pressures of Gambian children. Int J Epidemiol. 1991; 20, 938-943.

41. Ayoola OO, Gemmell I, Omotade OO, et al. Maternal malaria, birth size and blood pressure in Nigerian newborns: insights into the developmental origins of hypertension from the Ibadan growth cohort. PloS One. 2011; 6, e24548.

42. Hawkesworth S, Prentice AM, Fulford AJ, Moore SE. Maternal protein-energy supplementation does not affect adolescent blood pressure in the Gambia. Int J Epidemiol. 2009; 38, 119-127. 
43. Sadoh WE, Ibhanesehbor SE, Monguno AM, Gubler DJ. Predictors of newborn systolic blood pressure. West Afr J Med. 2010; 29, 86-90.

44. Nwokoye I, Uleanya N, Ibeziako N, et al. Blood pressure values in healthy term newborns at a tertiary health facility in Enugu, Nigeria. Niger J Clin Pract. 2015; 18, 584-588.

45. Silva ABT, Capingana DP, Magalhaes P, et al. Predictors and reference values of pulse wave velocity in prepubertal Angolan children. J Clin Hypertens. 2016; 18, 725-732.

46. Kagura J, Adair LS, Munthali RJ, Pettifor JM, Norris SA. Association between early life growth and blood pressure trajectories in black South African children. Hypertension. 2016; 68, 1123-1131.

47. Adair LS, Fall CH, Osmond C, et al. Associations of linear growth and relative weight gain during early life with adult health and human capital in countries of low and middle income: findings from five birth cohort studies. Lancet. 2013; 382, 525-534.

48. Griffiths PL, Sheppard ZA, Johnson W, et al. Associations between household and neighbourhood socioeconomic status and systolic blood pressure among urban South African adolescents. J Biosoc Sci. 2012; 44, 433-458.

49. Levitt NS, Steyn K, De Wet T, et al. An inverse relation between blood pressure and birth weight among 5 year old children from Soweto, South Africa. J Epidemiol Community Health. 1999; 53, 264-268.

50. Woelk G, Emanuel I, Weiss NS, Psaty BM. Birthweight and blood pressure among children in Harare, Zimbabwe. Arch Dis Child Fetal Neonatal Ed. 1998; 79, F119-F122.

51. Youmbissi TJ, Oudou N, Mbede J, Nasah BT. Blood pressure profiles of a group of African children in the first year of life. J Trop Pediatr. 1989; 35, 245-246.
52. Longo-Mbenza B, Ngiyulu R, Bayekula M, et al. Low birth weight and risk of hypertension in African school children. J Cardiovasc Risk. 1999; 6, 311-314.

53. Chiolero A, Paradis G, Madeleine G, et al. Birth weight, weight change, and blood pressure during childhood and adolescence: a school-based multiple cohort study. J Hypertens. 2011; 29, 1871-1879.

54. Salvi P, Meriem C, Temmar M, et al. Association of current weight and birth weight with blood pressure levels in Saharan and European teenager populations. Am J Hypertens. 2010; 23, 379-386.

55. Woelk GB. Is low birth weight a risk factor for adult hypertension? A literature review with particular reference to Africa. $S$ Afr Med J. 1995; 85(Pt 2), 1348-1349, 1352-1353.

56. Levitt NS, Lambert EV, Woods D, et al. Impaired glucose tolerance and elevated blood pressure in low birth weight, nonobese, young South African adults: early programming of cortisol axis. J Clin Endocrinol Metabol. 2000; 85, 4611-4618.

57. Berlin JA, Begg CB, Louis TA. An assessment of publication bias using a sample of published clinical trials. J Am Stat Assoc. 1989; 84, 381-392.

58. Ewald DR, Haldeman DL. Risk factors in adolescent hypertension. Glob Pediatr Health. 2016; 3, 2333794 X15625159.

59. Hardy R, Sovio U, King VJ, et al. Birthweight and blood pressure in five European birth cohort studies: an investigation of confounding factors. Eur J Public Health. 2006; 16, 21-30.

60. Huxley R, Neil A, Collins R. Unravelling the fetal origins hypothesis: is there really an inverse association between birthweight and subsequent blood pressure? Lancet. 2002; 360, 659-665.

61. Curhan GC, Willett WC, Rimm EB, et al. Birth weight and adult hypertension, diabetes mellitus, and obesity in US men. Circulation. 1996; 94, 3246-3250. 